\section{THE NEONATAL FC RECEPTOR IS ELEVATED IN MONOCYTE-DERIVED IMMUNE CELLS IN PANCREATIC CANCER}

Justin Thomas*, Molly Torok, Kriti Agrawal, Trang Vu, Alyssa Castillo, Min Chen, Bryan Remaily, Kyeongmin Kim, Zhiliang Xie, Samuel Kulp, Dwight Owen, Mitch Phelps, Christopher Coss, Thomas Mace. The Ohio State University, Columbus, OH, United States

Background Pancreatic ductal adenocarcinoma (PDAC) is the third leading cause of cancer-related death in the United States with 5 -year survival rates below $10 \%$. PDAC is commonly diagnosed after metastasis has occurred and treatment options are limited. Immune checkpoint inhibitor (ICI) monoclonal antibody $(\mathrm{mAb})$ therapy has shown great promise in other cancers, however little efficacy has been observed in patients with PDAC. The protein responsible for recycling IgG based $\mathrm{mAb}$ therapeutics like ICIs in the bloodstream, as well as processing peptides for antigen presentation, is the neonatal $\mathrm{Fc}$ receptor $(\mathrm{FcRn})$. Little is known about $\mathrm{FcRn}$ in cancer, and to our knowledge no characterization of host FcRn, or FcRn extrinsic to tumor cells exists in PDAC patients. We hypothesized that PDAC patients and tumor-bearing animals would have altered FcRn expression by their immune populations compared to their healthy counterparts.

Methods C57BL/6 mice were orthotopically injected with KPC-luc (KrasLSL-G12D, Trp53LSL-R270H, Pdx1-cre) pancreatic tumor cells, and FcRn expression in myeloid-derived splenocytes were analyzed by fluorescence cytometry. Time-offlight mass cytometry (CyTOF) was utilized to immunophenotype peripheral blood mononuclear cells (PBMCs) of PDAC or non-cancer patients for expression levels of FcRn within these immune populations.

Results PDAC tumor-bearing mice exhibit altered FcRn expression among myeloid immune cell populations. Mice with pancreatic tumors had elevated expression of $\mathrm{FcRn}$ on migratory cDC2 (CD8-CD11b+CD103+CD24++; p = 0.017), monocytic MDSC (CD11b+Ly6G-Ly6C+; p $=0.0023)$, granulocytic MDSC (CD11b+Ly6G+Ly6C $\pm ; p=0.0542)$, and cDC2 (CD8-CD11b+CD103-CD24 \pm ; $\mathrm{p}=0.036)$ cells. PBMCs from non-cancer obese patients (healthy control samples; $n=8$ ) and PDAC patients prior to surgical resection $(n=13)$ were subjected to CyTOF analyses. The majority of FcRn expression was concentrated to monocyte $(\mathrm{p}=0.017)$, DCs $(\mathrm{p}=0.017)$ and MDSC $(\mathrm{p}=0.012)$ immune populations. Overall, we observed increased expression of FcRn on myeloidderived immune populations from patients with PDAC. FcRn expression was elevated in both monocytes and DC populations in PDAC relative to non-cancer PBMCs. Monocytic and granulocytic MDSC from patients with PDAC had significantly elevated FcRn positivity compared to healthy controls $(\mathrm{p}=$ $0.034, \mathrm{p}=0.026$, respectively).

Conclusions FcRn is upregulated in monocytes, dendritic cells and MDSC immune populations in patients and mice with pancreatic tumors. Future investigations into FcRn function in preclinical models and PDAC patients will hopefully elucidate new mechanisms of ICI resistance and possible alternative approaches for improving immunotherapy efficacy in these patients.

Ethics Approval All patients provided voluntary written informed consent (Institutional Review Board protocol: 2010C0051) to participate. The protocols and subsequent amendments were approved by The Ohio State University Institutional Review Board. All animal protocols were approved by the Ohio State University Institutional Animal
Care and Use Committee (IACUC) at The Ohio State University (Approved IACUC protocols 2009A0178-R4 and 2017A00000117-R1) and mice were treated in accordance with institutional guidelines for animal care. The Ohio State University Laboratory Animal Shared Resource is an Association for Assessment and Accreditation of Laboratory Animal Care International accredited program that follows Public Health Service policy and guidelines. All other experiments were completed under the research protocols (2014R00000086; 2013R00000056) approved by the Ohio State University Institutional Biosafety Committee.

http://dx.doi.org/10.1136/jitc-2021-SITC2021.678 